Instituto Internacional de Investigación y Desarrollo Tecnológico Educativo INDTEC, C.A.

DOI: https://doi.org/10.29394/Scientific.issn.2542-2987.2019.4.14.13.271-285

OAI-PMH: http://www.indteca.com/ojs/index.php/Revista Scientific/oai

Artículo Original / Original Article

\title{
Habilidades Pedagógicas del profesor universitario como tutor mediador del servicio comunitario
}

\author{
Autores: Nellys Josefina Pitre Lugo \\ Universidad Nacional Experimental "Rafael María Baralt", UNERMB \\ nellyspitre77@hotmail.com \\ Altagracia, Venezuela \\ https://orcid.org/0000-0003-2032-7564 \\ Julio Rafael Pire Morales \\ Universidad Nacional Experimental "Rafael María Baralt", UNERMB \\ juliopire@hotmail.com \\ Altagracia, Venezuela \\ https://orcid.org/0000-0002-4674-887X \\ Daniela del Valle Díaz Piña \\ Universidad Nacional Experimental "Rafael María Baralt”, UNERMB \\ Icdadanieladiaz@gmail.com \\ Altagracia, Venezuela \\ https://orcid.org/0000-0003-0815-0970
}

\section{Resumen}

El presente estudio se realizó con el propósito de examinar las habilidades pedagógicas desarrolladas por el profesor universitario como tutor mediador del servicio comunitario. La investigación es de tipo analítica, diseño no experimental, de campo, transaccional. La población estuvo conformada por 10 profesores tutores de servicio comunitario y 50 estudiantes prestadores del servicio, programa Administración en la Universidad Nacional Experimental "Rafael María Baralt" (UNERMB), sede Los Puertos. La técnica de recolección de datos fue la observación y un cuestionario con 45 ítems y cinco (5) alternativas de respuesta, el cual fue validado a través del juicio de cinco (5) expertos, la confiabilidad se determinó mediante el coeficiente de Alfa Cronbach arrojando un 98,9\%. Los resultados indicaron que los docentes no poseen las habilidades pedagógicas requeridas para el desarrollo exitoso del servicio comunitario, pese a que algunos casos articulan las actividades y logran en cierta medida la dirección de líderes comunitarios, se consideró debido a los porcentajes obtenidos que no es suficiente para garantizar la presencia de las habilidades asociadas al diseño e implementación de estrategias de aprendizaje que conlleven al logro de los objetivos del servicio comunitario.

Palabras clave: comunidad; pedagogía; profesor; tutoría; universidad.

Cómo citar este artículo:

Pitre, N., Pire, J., \& Díaz, D. (2019). Habilidades Pedagógicas del profesor universitario como tutor mediador del servicio comunitario. Revista Scientific, 4(14), 271-285, e-ISSN: 2542-2987. Recuperado de: https://doi.org/10.29394/Scientific.issn.2542-2987.2019.4.14.13.271-285

Fecha de Recepción: 27-05-2019
Fecha de Aceptación: 03-08-2019
Fecha de Publicación: 05-11-2019 


\title{
Pedagogical skills of the university professor as a mediator tutor of the community service
}

\begin{abstract}
The present study was carried out with the purpose of examining the pedagogical skills developed by the university professor as a mediator tutor of the community service. The research is analytical, non-experimental, field, transactional. The population was made up of 10 community service tutors and 50 service providers, Administration program at the "Rafael María Baralt" National Experimental University (UNERMB), Los Puertos headquarters. The data collection technique was the observation and a questionnaire with 45 items and five (5) response alternatives, which was validated through the judgment of five (5) experts, the reliability was determined by the alpha Cronbach coefficient throwing $98.9 \%$ The results indicated that teachers do not possess the pedagogical skills required for the successful development of community service, although some cases articulate the activities and achieve the leadership of community leaders to some extent, it was considered due to the percentages obtained that it is not enough to ensure the presence of the skills associated with the design and implementation of learning strategies that lead to the achievement of the objectives of the community service.
\end{abstract}

Keywords: communities; pedagogy; teacher; tutoring; universities.

How to cite this article:

Pitre, N., Pire, J., \& Díaz, D. (2019). Pedagogical skills of the university professor as a mediator tutor of the community service. Revista Scientific, 4(14), 271-285, e-ISSN: 2542-2987. Recovered from: https://doi.org/10.29394/Scientific.issn.2542-2987.2019.4.14.13.271-285

Date Received:

27-05-2019
Date Acceptance:

03-08-2019
Date Publication:

05-11-2019 


\section{Introducción}

Las instituciones universitarias desarrollan y aplican programas educativos con el propósito de formar profesionales con ética, afianzados en valores y con conocimientos técnicos, humanísticos y científicos, capaces de transformar la sociedad y transitar por el camino de la pluralidad, sin descuidar sus objetivos personales.

Para Terán y Araujo (2016), se destacan que:

Los organismos educativos sobresalen por su función modeladora de individuos que se integrarán y convivirán en las sociedades; de manera especial hay que mencionar las universidades, las cuales poseen los recursos técnicos y gnoseológicos para mejorar las condiciones de vida de la colectividad" (pág. 57).

Cabe enfatizar que la Ley de Universidades (1970), en su artículo 1, reza que: "la universidad es fundamentalmente una comunidad de intereses espirituales que reúne a profesores y estudiantes en la tarea de buscar la verdad y afianzar los valores trascendentales del hombre" (pág. 3). En este sentido es necesario que los docentes posean el perfil idóneo para administrar su cátedra, pero además es importante que den cumplimiento sus funciones de docencia, investigación y extensión, además de atender su responsabilidad como tutor de servicio comunitario, la cual permitirá fortalecer los valores comunitarios en los estudiantes de pregrado.

Lo anteriormente expuesto, implica el conocimiento de la Ley de Servicio Comunitario del Estudiante de Educación Superior (LSCEES), la cual lo define como una actividad que deben realizar los estudiantes de educación superior en las comunidades, con el fin de aplicar los conocimientos adquiridos durante la carrera, todo en función de cooperar con el bienestar social. Para que esto se cumpla es fundamental el apoyo del docente, es por ello que la presente investigación pretende analizar el rol del profesor en su faceta como tutor de servicio comunitario. 
La educación tiene la doble finalidad, de preparar a las personas para enfrentarse a la vida en procesos cambiantes, vertiginosos y tremendamente palpables; así como, convertirlos en fuentes de progreso, desarrollo y transformaciones sociales que le permitan mejorar su condición humana. En efecto, en el artículo 1 el cual contempla las Misiones y Funciones de la Educación Superior según la Organización Internacional para la Educación, la Ciencia y la Cultura (UNESCO, 1998): indica que tiene como misión formar profesionales altamente capacitados, ciudadanos responsables, competentes y comprometidos con el desarrollo social, alejados de postulados centrados en enseñanzas tradicionales, en la atención del docente como transmisor de conocimientos y valores reproducidos por los estudiantes (pág. 1).

Con base a esa referencia, se inicia la exposición con postulados que hablan del necesario protagonismo que los estudiantes universitarios han de tener ante currículos integrados al saber comunitario, asumiendo como problemática el planteamiento de Figueroa (2006): quien revela procesos de enseñanza en las instituciones de educación superior caracterizados por complejos e imprevisibles eventos que indican reformas en la labor cumplida por los profesores, evidenciando el reconocimiento de una tarea compleja cuando éstas hacen referencia a la formación de educandos como ciudadanos responsables, competentes y comprometidos con la sociedad actual, al igual sucede cuando se trata de una nueva concepción del docente universitario el cual requiere involucrarse en procesos de acompañamiento estudiantil (pág. $55)$.

Tal es el caso venezolano, en el cual a partir de la implementación de la Ley de Servicio Comunitario del Estudiante de Educación Superior en el año 2005, se convierte en un tema de gran interés para la formación universitaria, en tanto la ley busca potenciar prácticas y el modelaje de valores en un currículo contextualizado, flexible, para la producción social, incorporando profesionales y estudiantes críticos al saber comunitario, esto se asocia a lo 
planteado por González (2004), quien asegura que: "la concepción del profesor como persona que acompaña al estudiante en el proceso de enseñanza- aprendizaje exige nuevas funciones que se expresan en la consideración del profesor como modelo educativo" (pág. 1).

En consecuencia, puede decirse que la labor tutorial del docente universitario será la de un experto en materia comunitaria con dominio sobre los conocimientos adquiridos por cada asignatura administrada, normativas propuestas, reformas y cambios gestados en el ámbito universitario. Es decir, una labor superior a la gestión de información para actuar como transmisor de conocimientos, compartirlos y vivenciarlos en experiencias enriquecedoras, para lo cual han de desarrollarse debates reflexivos sobre contenidos de enseñanza en ambientes democráticos motivadores para desarrollar actitudes positivas con pertinencia en el entorno social.

Por lo tanto, no es suficiente contar con un docente universitario conocedor de la materia, es necesaria una formación pedagógica que posibilite la utilización de metodologías para el aprendizaje mediador, idoneidad para estimular a los estudiantes, disposición e interés como prestadores del servicio comunitario. Es necesario profesores que entiendan la tutoría del servicio comunitario como una función intrínseca de la función docente regulada por la legislación educativa insertándolos (con suficiente disposición), hacia el desarrollo de proyectos comunitarios significativos, con conocimientos sobre su área de actuación y los diferentes procedimientos requeridos para cumplir satisfactoriamente con el trabajo comunitario.

Ahora bien, en el nuevo contexto educativo latinoamericano, puede señalarse que en Venezuela el Ministerio de Educación Universitario ha generado importantes y novedosas estrategias para el desarrollo del sector educativo presumiéndose una estructuración del mismo con miras a elevar la calidad académica de estas instituciones, mejorar la calidad de acceso y desempeño estudiantil para lograr una mayor pertenencia social con las 
mismas, vincularse con distintos sectores de la sociedad, sobre todo promoviendo y fortaleciendo la participación comunitaria plasmada por el Ministerio del Poder Popular para alcanzar cooperaciones permanentes entre estas instituciones y las comunidades donde se insertan apoyando continuamente los espacios locales.

Tal iniciativa pudiera suscribirse en Venezuela a formar estudiantes con actitudes, disposición y compromiso social, profesores con capacidad para contribuir desde el seno universitario con el fortalecimiento de valores sobre calidad, equidad social y cooperación, es decir hacia una participación activa, protagónica y corresponsable con el entorno sobrepasando la educación tradicional que pareciera haber imperado en las aulas universitarias durante muchísimo tiempo.

Sin embargo, llevar a cabo la toma de decisiones sobre estos aspectos pudiera ser algo complejo, dado que acercarse al cumplimiento de la Ley de Servicio Comunitario desde la universidad se ha convertido en un aparente problema para los profesores quienes muchas veces se niegan a asumir la responsabilidad tutorial, alegando su alto compromiso por grandes cantidad de tareas para cumplir la función de docencia e investigación, así como dificultades financieras para responder satisfactoriamente con los recursos requeridos para la movilización organizada del servicio comunitario.

De acuerdo con lo anterior se demuestra que el éxito de la participación social enunciada mediante la ley de servicio comunitario para el estudiante universitario dependerá del rol que como tutor realice el docente para lograr actitudes favorables hacia este propósito. Sobre el particular, es oportuno apuntar que las evidencias anteriores revelan poca disposición del profesor para alcanzar éxitos en la labor tutorial, ya que la sistematización de esta práctica requiere orientación pedagógica en el área comunitaria y actualmente evidencia la ausencia de planes formales sobre aspectos teóricos prácticos dispuestos en la ley. Con ese planteamiento, queda reflejado que el tutor debe 
ser el nexo para unificar e integrar estudiantes a las comunidades.

Por otra parte, Ramírez (2005), el conjunto de habilidades pedagógicas del docente, implican actividades para:

a. Apoyarse en una planificación curricular donde se alineen los objetivos curriculares del servicio; b. Dar acompañamiento, crear condiciones entre docentes instrucciones de servicio público comunitario; c. Acompañar, motivar el desarrollo de los cursos; incorporando metodologías; d. Fomentar el reconocimiento y los incentivos para el desarrollo de iniciativas; e. Promover el fomento al desarrollo de la docencia para que profesores desarrollen cursos de servicio comunitario; $f$. Promover la investigación, la generación de conocimientos y la planificación de calidad en torno al servicio comunitario; g. Fomentar el desarrollo e impacto de las iniciativas en los distintos actores participantes; $h$. Desarrollar publicaciones que profundice en los aspectos del servicio comunitario; i. Velar por el cumplimiento del servicio comunitario; j. Crear un sistema de monitoreo que permita la retroalimentación del servicio comunitario; k. Fomentar el establecimiento de redes universitarias que permitan compartir aprendizajes y experiencias (pág. 56).

Lo referido por el autor evidencia que las habilidades de los profesores universitarios son necesarias para lograr procesos humanos integradores comunitarios, conocimientos y experiencias que redunden en su capacidad para planear, coordinar, ejecutar actividades de servicio comunitario en forma sistemática y efectiva.

El objetivo de la investigación está referido a examinar las habilidades pedagógicas desarrolladas por el profesor universitario como tutor mediador del servicio comunitario.

\section{Metodología}

La presente investigación se catalogó como analítica de enfoque proyectivo, utilizando el método de investigación cuantitativo (positivista), de acuerdo con sus características metodológicas corresponde a un diseño no 
experimental de campo documental, transversal. Se trata de una investigación de campo, debido a que los datos serán obtenidos en el sitio en el cual por naturalidad lo hechos se suceden de forma cotidiana, en este caso particular en la Universidad Nacional Experimental "Rafael María Baralt", (UNERMB), sede Los Puertos de Altagracia.

En esta ocasión la población objeto de estudio está comprendida por dos grupos, uno por el conjunto de Profesores que laboran en el Programa Administración de la Universidad Nacional Experimental "Rafael María Baralt", que fungen como tutores y 50 estudiantes cursantes del servicio comunitario.

La forma en que los investigadores asumieron la selección de la misma fue a través de un censo, por cuanto se acudió a la revisión de las nóminas de personal docente de la Secretaria de dicho Programa y con la Coordinación de Servicio Comunitario así como a las planillas de registros de los estudiantes cursantes del servicio comunitario.

Para orientar la búsqueda de recolección de información necesaria para profundizar en la problemática estudiada se procedió a utilizar técnicas de observación directa a los profesores, la técnica utilizada fue la encuesta mediante la aplicación de un cuestionario, para tal fin se elaboró un cuestionario con alternativas de respuestas: Frecuentemente (5), A menudo (4), A veces (3), Rara vez (2) y nunca (1), es decir en una escala de actitudes.

Una vez elaborado el instrumento para la recolección de datos, se sometió a la comprobación de dos aspectos fundamentales la validez y la confiabilidad. En efecto la validez del contenido de los cuestionarios se realizó a través del juicio de cinco (5) expertos, doctores en Ciencias de la Educación y especialistas en el área de investigación, a quienes se les solicito el apoyo y consideración respectiva.

Luego de haber obtenido la validez del contenido de los instrumentos se aplicó una prueba piloto a veinte (20) sujetos. Esta prueba fue necesaria para calcular la confiabilidad del mismo. La confiabilidad se calculó utilizando 
el coeficiente de Alfa de Cronbach, empleando para ello el software estadístico SPSS, Versión 20.0, para Ciencias Sociales, el cual arrojó un 98,8\%

Para la interpretación y análisis de los datos se construyó un baremo a propósito de realizar el análisis basado en el método de la estadística descriptiva procesada en relación a técnicas de relación porcentual (\%); para expresar los aspectos valorados en categorías el cual se encuentra detallado en la tabla 1.

Tabla 1. Baremo para el análisis de los resultados.

\begin{tabular}{|c|c|c|}
\hline Escala & $\begin{array}{c}\text { Escala } \\
\text { (Valoración cualitativa) }\end{array}$ & Porcentaje (\%) \\
\hline Frecuentemente (F) & Totalmente Eficiente & $81-100$ \\
\hline A Menudo (AM) & Eficiencia Aceptable & $61-80$ \\
\hline A veces (AV) & Medianamente Eficiente & $41-60$ \\
\hline Rara Vez (RV) & Deficiente & $21-40$ \\
\hline Nunca (N) & Totalmente Deficiente & $0-20$ \\
\hline
\end{tabular}

Fuente: Los Autores (2019).

\section{Procedimiento de la Investigación}

1. Se seleccionó el tema, tomando en cuenta la necesidad de los investigadores de realizar un estudio sobre el rol desempeñado por el profesor universitario como tutor del servicio comunitario.

2. Se indagó la bibliografía relacionada con el estudio y los antecedentes que sirvieron como punto de referencia para que la presente investigación se lleve a cabo.

3. Se elaboró el instrumento pertinente, el cual fue sometido al proceso de validez y confiabilidad.

4. Se aplicó el instrumento inicialmente en la prueba piloto y posteriormente a la población objeto de estudio.

5. Los resultados obtenidos se codificaron y tabularon, los cuales fueron analizados e interpretados. 
6. Con base a los resultados obtenidos, se elaboraron las conclusiones.

7. Posteriormente se realizaron las recomendaciones que surgieron luego del cotejo entre las conclusiones y los objetivos específicos de esta investigación.

8. Finalmente se realizó un plan de acciones pedagógicas.

Tabla 2. Resultados.

\begin{tabular}{|c|c|c|c|c|}
\hline Indicador & $\%$ & Interpretación & Promedio & Interpretación \\
\hline $\begin{array}{c}\text { Articulación de } \\
\text { las actividades } \\
\text { del Servicio } \\
\text { Comunitario al } \\
\text { plan de estudio. }\end{array}$ & 43,33 & $\begin{array}{c}\text { MEDIANAMENTE } \\
\text { EFICIENTE }\end{array}$ & & \multirow{2}{*}{ DEFICIENTE } \\
$\begin{array}{c}\text { Aplicación de } \\
\text { estrategias } \\
\text { instruccionales }\end{array}$ & 30,00 & DEFICIENTE & 40,00 & \\
\hline $\begin{array}{c}\text { Dirección de } \\
\text { líderes para el } \\
\text { trabajo }\end{array}$ & 46,67 & $\begin{array}{c}\text { MEDIANAMENTE } \\
\text { EFICIENTE }\end{array}$ & & \\
\hline
\end{tabular}

\section{Conclusiones}

Los resultados presentados en la tabla 2, permitieron desarrollar conclusiones en cuanto las habilidades pedagógicas desarrolladas por el profesor universitario como tutor mediador del servicio comunitario, en lo que respecta a la articulación de las actividades del servicio comunitario al plan de estudio, se determinó que el docente en algunas ocasiones relaciona los contenidos de su asignatura con los propósitos que establece legalmente el servicio comunitario, algunas veces exterioriza sus experiencias de servicio comunitario en las cátedras que imparte e intercambia dichas prácticas con profesores de otras asignaturas.

Gracias a la información suministrada por ambas poblaciones (docentes 
y estudiantes), se pudo comprobar que el rol del docente en este aspecto es deficiente, es decir, el tutor no posee las habilidades requeridas para el desarrollo exitoso del servicio comunitario, pese a que algunos casos articulan las actividades y logran en cierta medida la dirección de líderes comunitarios, se consideró debido a los porcentajes obtenidos que no es suficiente para garantizar la presencia de las habilidades asociadas al diseño e implementación de estrategias de aprendizaje que conlleven al logro de los objetivos del servicio comunitario.

Es evidente entonces, que el profesor para ejecutar su rol de tutor mediador amerita la integración de conocimientos, estrategias didácticas y el intercambio de experiencias que fomenten la empatía y el deseo de los estudiantes de incorporarse a las comunidades y contribuir en la búsqueda de soluciones a los problemas que les aqueja.

\section{Referencias}

Figueroa, L. (2006). Formación docente: Complejidad y profesionalización. ETHOS Educativos, 35, 19-39, e-ISSN: 14057255. Recuperado de: http://imced.edu.mx/Ethos/Archivo/35-19.htm González, V. (2004). El profesorado universitario: su concepción y formación como modelo de actuación ética y profesional. Revista Iberoamericana De Educación, 34(1), 1-11, ISSN: 1022-6508; e-ISSN: 1681-5653. Recuperado de: https://rieoei.org/RIE/article/view/2934

Ley de Universidades (1970). Título I. Artículo 1. Gaceta Oficial No. 1429, Extraordinario, del 8 de septiembre. Caracas, Venezuela: Consejo Nacional de Universidades. Recuperado de:

http://www.unimet.edu.ve/unimetsite/wp-content/uploads/2013/02/Leyde-Universidades-1970.pdf

Ramírez, T., \& Pizarro, B. (2005). Aprendizaje Servicio: Manual para Docente U.C. Santiago, Chile: Pontificia Universidad Católica de Chile. 
Recuperado de: http://www.zerbikas.es/producto/aprendizaje-serviciomanual-para-docentes-uc/

\section{UNESCO (1998). Declaración Mundial sobre la Educación Superior en el} siglo XXI: Visión y Acción. Conferencia Mundial sobre la Educación Superior en el siglo XXI: Visión y acción, Código del documento: ED.98/CONF.202/3. París, Francia: Organización de las Naciones Unidas para la Educación, la Ciencia y la Cultura. Recuperado de: http://www.unesco.org/education/educprog/wche/declaration spa.htm Terán, C., \& Araujo, W. (2016). El Servicio Comunitario, Una Mirada Teórica. Revista Scientific, 1(2), 54-74, e-ISSN: 2542-2987. Recuperado de:

https://doi.org/10.29394/scientific.issn.2542-2987.2016.1.2.4.54-74 


\section{Nellys Josefina Pitre Lugo}

e-mail: nellyspitre77@@hotmail.com 


\section{Julio Rafael Pire Morales}

e-mail: juliopire@hotmail.com

Nacido en Carirubana, estado Falcón, Venezuela, el 18

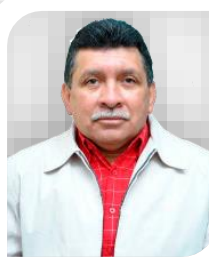
de febrero del año 1957. Doctor en Ciencias Gerenciales; Magister en Gerencia de Empresas, mención Gerencia de Mercadeo; Ingeniero Industrial, egresado de la Universidad del Zulia (LUZ); Docente Universitario, categoría Asociado en el programa Administración en la Universidad Nacional Experimental "Rafael María Baralt" (UNERMB), con 23 años de experiencia, administrando las cátedras Matemáticas, Métodos Cuantitativos, Toma de Decisiones, Estrategia y Política Gerencial; Profesor invitado en Postgrado de la Universidad Nacional Experimental Politécnica de la Fuerza Armada Bolivariana (UNEFA) y la Universidad Privada Dr. Rafael Belloso Chacín (URBE); Tutor de tesis, pasantías y servicio comunitario; Experiencia en docencia virtual y en la industria petrolera; Acreditado como Investigador A en el Programa de Estímulo a la Innovación e Investigación (PEII). 
Nacida en Coro, estado Falcón, Venezuela, el 14 de noviembre del año 1987. Magister Scientiarum en Gerencia Financiera; Licenciada en Administración, egresada de la Universidad Nacional Experimental "Rafael María Baralt" (UNERMB); con Diplomados en Gerencia de RRHH (CEFIMAR) y Administración Tributaria de la Universidad Privada Dr. Rafael Belloso Chacín (URBE); Componente Docente Nivel 9 de Inglés en el Centro Venezolano Americano del Zulia (CEVAZ); Docente Universitario, categoría Asistente en el programa Administración en la UNERMB; Cátedras Gestión de Personal, Estructura Organizacional y Planificación Tributaria; Tutora de tesis, pasantías y servicio comunitario. Experiencia en docencia virtual y cargos gerenciales. 